\title{
Virtual Screening, Molecular Docking, and ADME/T Analysis of Natural Product Library against Cell Invasion Protein SipB from Salmonella enterica serotype typhi: In Silico Analysis
}

\author{
Aminu Ibrahim Danyaya ${ }^{1 \#}$, Abhishek Kumar Verma ${ }^{3 \# *}$, Avinash \\ Kumar $^{2 \#, ~ B i n t a ~ S u n u s i ~ S h u a i b u ' ~}{ }^{1}$, Umar Adamu Hamza ${ }^{1}$, Najib Lawan \\ Yahaya $^{1}$, Usman Rabi'u Bello ${ }^{1}$, Zaharaddeen Umar Na'abba ${ }^{1}$ and \\ Abubakar Dabo Dalhat ${ }^{1}$ \\ ${ }^{1}$ Department of Life Sciences, School of Science and Technology, Mewar University, \\ Chittorgarh, Rajasthan, India \\ ${ }^{2}$ Department of Paramedical Sciences, Mewar University, Chittorgarh, Rajasthan, \\ India \\ ${ }^{3}$ Assistant Professor, Department of Biochemistry, Mewar University, Chittorgarh, \\ Rajasthan, India \\ ${ }^{\#}$ Authors Contributed Equally \\ *Corresponding Author: Abhishek Kumar Verma, Assistant Professor, Department \\ of Biochemistry, Mewar University, Chittorgarh, Rajasthan, India.
}

DOI: $10.31080 /$ ASPS.2020.04.0563
Received: July 01, 2020

Published: July 18, 2020

(C) All rights are reserved by Abhishek Kumar Verma., et al.

\begin{abstract}
Background: The most dangerous issue in the healthcare arises is outbreak of the antibiotic-resistant bacteria worldwide. The randomness of Salmonella infections rely on its serotypes and the immune response of the host. Children up to age of 5 years and patients of older age are prone to the salmonella infections. The virulence genes of Salmonella encodes five different groups of Cell invasion proteins (CIPs), including Sip A, B, C, D and E. Upon contact with the target cell, SipB undergo 'type III' export from the bacterial cytoplasm and translocate into the cell membrane. Caspase-1 activity is essential for the cytotoxicity, and it has been proven that binding of SipB to caspase-1 induces macrophage apoptosis, so functional inhibition of caspase-1 blocks macrophage cytotoxicity.

Methods: This study is to determine the components of the natural origin compounds (NOCs) have an anti-bacterial effect (ABE) and capable to prevent the humans from bacterial infection Salmonella enterica serotype typhi. This Salmonella is using the most reliable method is suitable for molecular docking. We used to find out the interaction study between the molecules and the protein. In our study based on the inhibitor of Cell Invasion Protein SipB from Salmonella. We performed In Silico method for screening of all the natural compounds against Cell Invasion Protein SipB inhibition.

Results and Discussion: The results we obtained from molecular docking shown that among 2228 molecules of natural origin from natural product compounds library (20200427-L1400) was retrieved in SDF format from Inhibitor Expert (Selleckchem.com). Fifteen molecules are the best compounds observed through molecular docking and hydrophobic interactions and hydrogen bonding with interaction are proposed as the novel inhibitors against the Cell Invasion Protein SipB from Salmonella. We demonstrate using SwissADME online server tools and DruLito that all fifteen natural molecules has better "drug-likeness and does not violate any Lipinski, Ghose, Veber, Egan or Muegge rules.

Conclusion: Importantly, all fifteen natural compounds are more potent in treatment of against Cell Invasion Protein SipB in Salmonella typhi but needs further experimental research.
\end{abstract}

Keywords: Salmonella enterica; Serotype typhi; Cell Invasion Protein SipB; Natural Product Library; Molecular Docking 
Highlights

- $\quad$ Cell Invasion Protein SipB of Salmonella enterica serotype typhi bind with selected natural compounds from Inhibitor Expert (Selleckchem.com).

- $\quad$ Cell Invasion Protein SipB interacted with drugs by hydrophobic interactions and hydrogen bond.

- Natural compounds show good binding free energy with protein complexes.

- Ligands were found to follow the Lipinski rule of five.

\section{Introduction}

The spread of antibiotic-resistant bacteria stands as one of the most dangerous health care issues to human health worldwide, with Food of animal origin such as eggs, milk, poultry, beef and pork contributing to a large extent for zoonotic transmission of the non-typhoidal Salmonella serotype [1,2]. In humans, the severity of Salmonella infections depends on the serotypes involved and the health condition of the host. Young children under the age of 5 years, old people and patients whose immune system is suppressed are more prone to the infection than healthy individuals $[3,4]$.

The most common sign of Salmonella infection in healthy adults is Gastroenteritis, then bacteremia and enteric fever follows [5,6], whereas systemic disease can also happen in children, elderly people and those with compromised immune system [7].

Salmonella infection do not only affect humans, as animal too are not exempted, be it mammals, birds or other cold-blooded animals [8]. But it is more common in poultry and swine as compared to cattle. Fowl typhoid and pullorum are septicemic diseases that mostly occur in chickens and turkeys, with Salmonella gallinarum and $S$. pullorum, being the causative agents respectively. Anorexia, diarrhea, dehydration, weakness and high mortality are the clinical signs of the diseases in chicks and poults. More so, decreased in egg production, fertility and hatchability problems are the common signs of Salmonellosis in mature fowls $[9,10]$.

The genus Salmonella is a gram negative, rod shaped and motile bacteria that is serologically divided into over 2500 serovars within the enterica species. Many of the $S$. enterica serovars actively invade the mucosal surface of the intestine and $S$. typhi has evolved the ability to spread deeper into human tissues such as spleen, liver and bone marrow [11-13]. Large number of virulence genes play a special role in the colonization of hosts by $S$. enterica, and molecular evaluation shows that most of these virulence genes are clustered within Salmonella Pathogenicity Islands (SPI). The Salmonella enterica serovar typhi has numerous Pathogenicity Island which controls its adhesion and colonization, invasion, intracellular survival and proliferation, so understanding the detailed mechanism for survival of this organism in the host cell is significant for effective detection, treatment and drug design of this pathogen.

The virulence genes of Salmonella encodes five different groups of Cell invasion proteins (Sips), including Sip A, B, C, D and E [14]. Upon contact with the target cell, SipB undergo 'type III' export from the bacterial cytoplasm and translocate in to the cell membrane. Caspase- 1 activity is essential for the cytotoxicity, and it has been proven that binding of SipB to caspase-1 induces macrophage apoptosis, so functional inhibition of caspase-1 blocks macrophage cytotoxicity [15]. Medicinal plants were the major source of medicines over many centuries. We have many examples of plant-derived extracts and/or compounds isolated from plants that have been widely used in the treatment of many significant diseases. To name just two examples, Turmeric (Curcuma longa) and Congress Grass (Parthenium hysterophorus L.) have been used over a long period of time. In 1806 Friedrich Serturner isolated the alkaloid morphine from poppy: an event that prompted a continuing search for other plant-derived medicines. Almost Natural compounds show the good anticancer, anti-bacterial, and anti-viral property [16].

\section{Materials and Methods}

\section{Protein preparation}

The crystal structure of Cell invasion protein SipB (PDB entry code: 6TUL) was downloaded from the protein databank (http:// www.rcsb.org), and its original ligand and water were eliminated, then all compounds from our data set were docked in the active site of the studied protein. The preparation of the PDB file was done using Discovery Studio 2016 [17].

\section{Library preparation}

The literature-based 3D or 2D structure of natural product compounds library (20200427-L1400) was retrieved in SDF format from Inhibitor Expert (Selleckchem.com). All the unique collection of 2228 natural products for high throughput screening, high content screening, Structurally diverse, bioactive, cell permeable, Rich documentation with structure, NMR, and High Performance Liquid Chromatography validated ensure high purity. Open Babel molecule format converter was used for the conversion of $2 \mathrm{D}$ to $3 \mathrm{D}$ conformation and Marvin Sketch software (version 15.10.0) per- 
Virtual Screening, Molecular Docking, and ADME/T Analysis of Natural Product Library against Cell Invasion Protein SipB from Salmonella enterica serotype typhi: In Silico Analysis

formed the conversion from SDF to pdf (for docking) and mol (for molecular properties prediction) file. Ligand's energy was minimized by relating the mmff94 force field and conjugate gradients optimization algorithm using PyRx- Python prescription 0.8.

Visual screening and molecular docking

Molecular docking analysis was used to study the binding affinity and the type of interactions between all compounds and the target Salmonella Cell inversion protein (SipB). The steps for preparing ligands and proteins for docking protocol were done in the Autodock 1.5.4 tools from MGL Tools package employing default settings [18], a grid box (for center x -2.4236, y 2.3899, z 21.2142 and Dimensions (Angstrom) x 84.8047, Y 63.4355 and Z 59.4975), the bioactive conformations were simulated employing Autodock Vina. For Auto Dock Vina study, an extended PDB format, termed PDBQT, is used for coordinate files, which includes atomic partial charges and atom types. Torsion angles were calculated to assign the flexible and non-bonded rotation of molecules. The results were subsequently analyzed using Discovery studio 2016 and PyMOL.

\section{ADMET properties}

For the estimation of the ADMET properties, a statistical analysis of natural compounds was carried out. In this analysis, we evaluated ADMET properties using the ADMET predictor FAFDrugs2 that runs on Linux OS. This method is freely available and used for filtering in Silico ADMET. This technique has been widely used as a screen for compounds that are expected to be produced more for product design programs. We have tested parameters such as the number of rotatable No. bonds ( $>10)$ and the number of rigid bonds that suggest good oral bioavailability and good intestinal absorption of the compound.

\section{Results and Discussion}

Analysis of docking results of promising compounds

The Crystal structure of the Cell invasion protein SipB (PDB: 6TUL) of Salmonella Typhi was used for docking purposes. Molecular docking has been performed using the extra precision (XP) mode of grid based ligand docking with energetics. We used known natural compounds for docking purpose (Figure 1-3). We selected the top 15 compounds with show good binding affinity, which were docked with Cell Invasion protein SipB of Salmonella Typhi. Our result highlighted that; Cyclocurcumin; Stigmasterol; Procurcumenol; 1,7-bis (4-hydroxyphenyl)-1,4,6-heptatrien-3-one; Curcumenol; 1,5-Bis(4-hydroxyphenyl)penta-1,4-dien-3-one; (1E,4E)-1-(4-Hydroxy-3-methoxyphenyl)-5-(4-hydroxyphenyl)-1,4-pentadiene-3one; 2-Hepten-4-one, 6-(3-hydroxy-4-methylphenyl)-2-methyl-; (6Z, 10S)-6, 10-Dimethyl-3-propan-2-ylidenecyclodec-6-ene-1, 4-Dione; Epiprocurcumenol; Cubebene; Isoprocurcumenol; BetaCaryophyllene; 1,3-dihydroxy-5-(tridec-4',7'-dienyl)benzene and DL-Borneol yielded a preeminent dock score with the Cell Invasion protein SipB with docking score of -8.8, -8.8, -8.0, -7.8, -7.7, -7.6, $-7.5,-7.3,-7.1,-7.1,-6.6,-6.6,-6.5,-6.3$ and $-5.6 \mathrm{Kcal} / \mathrm{mol}$ respectively (Table 1).

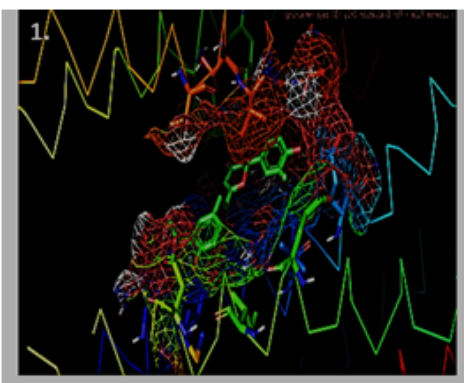

6TUL- Cyclocurcumin

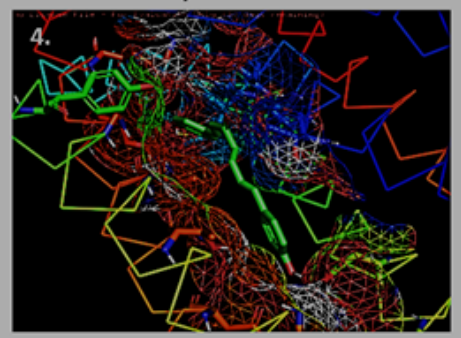

6TUL-1,7-bis (4-hydroxyphenyl)-1,4,6heptatrien-3-one

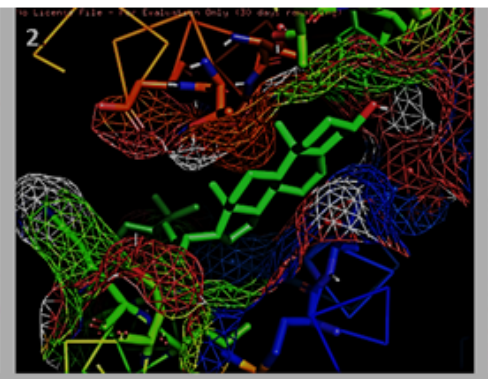

6TUL-Stigmasterol

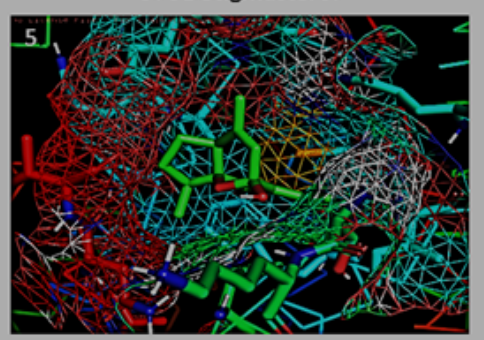

6TUL-Curcumenol

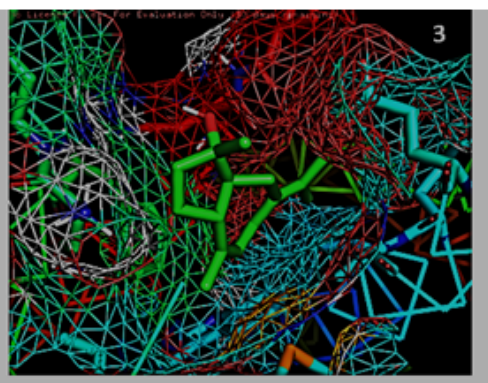

6TUL-Procurcumenol

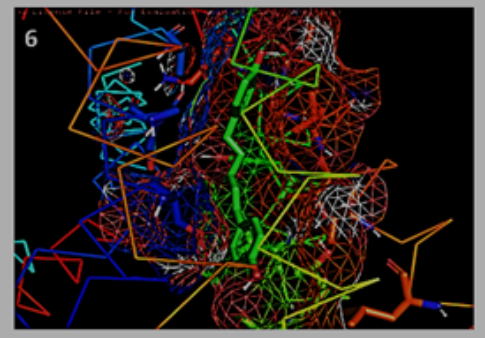

6TUL-1,5-Bis(4-hydroxyphenyl)penta-1,4dien-3-one

Figure 1: Mesh presentation of Cell invasion protein SipB (PDB: 6TUL) with top selected compounds. 1. Cyclocurcumin-Cell invasion protein SipB; 2. Stigmasterol- Cell invasion protein SipB; 3.Procurcumenol- Cell invasion protein SipB; 4. 1, 7-bis (4-hydroxyphenyl)-1, 4, 6-heptatrien-3-one- Cell invasion protein SipB 5. Curcumenol- Cell invasion protein SipB 6. 1, 5-Bis (4-hydroxyphenyl) penta-1, 4-dien3-one-Cell invasion protein SipB. 


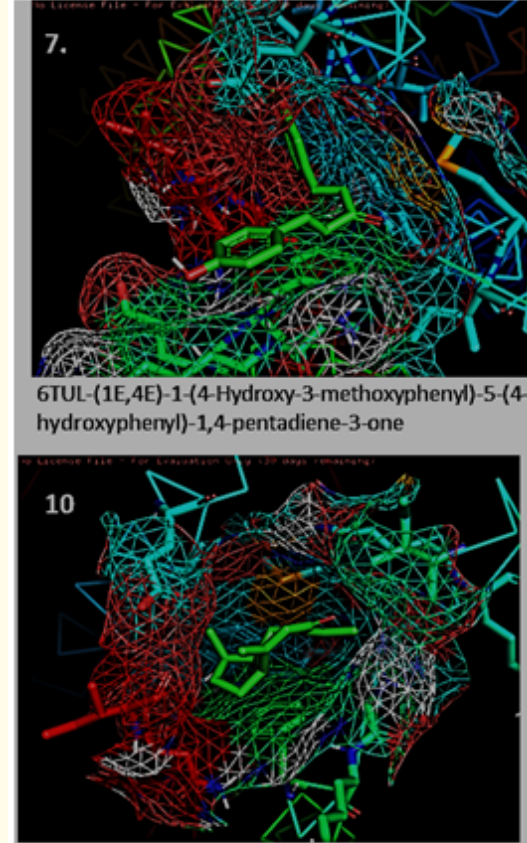

6TUL-Epiprocurcumenol

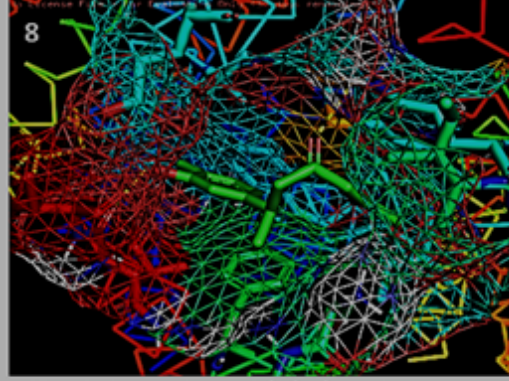

6TUL-2-Hepten-4-one, 6-(3-hydroxy-4 methylphenyl)-2-methyl-

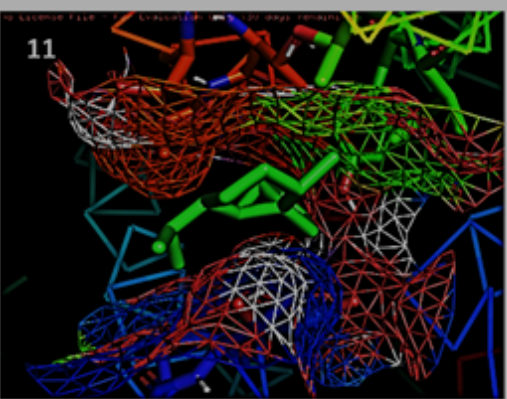

6TUL-Cubebene

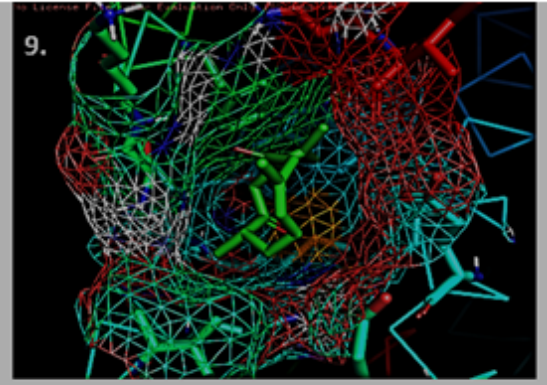

6TUL-(6Z, 10S)-6, 10-Dimethyl-3-propan-2ylidenecyclodec-6-ene-1, 4-Dione

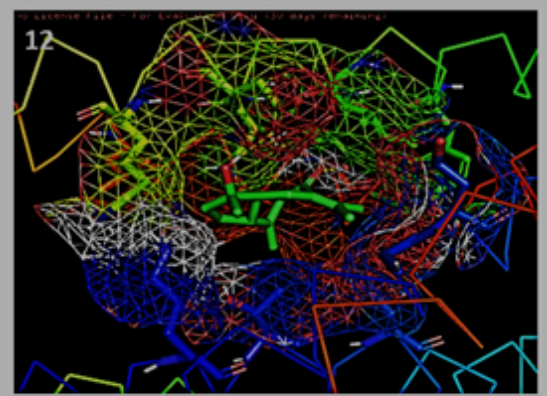

6TUL-Isoprocurcumenol

Figure 2: Mesh presentation of Cell invasion protein SipB (PDB: 6TUL) with top selected compounds. 7. (1E, 4E)-1-(4-Hydroxy-

3-methoxyphenyl)-5-(4-hydroxyphenyl)-1, 4-pentadiene-3-one-Cell invasion protein SipB; 8. 2-Hepten-4-one, 6-(3-hydroxy4-methylphenyl)-2-methyl- Cell invasion protein SipB; 9.6Z, 10S)-6, 10-Dimethyl-3-propan-2-ylidenecyclodec-6-ene-1, 4-Dione- Cell invasion protein SipB 10. Epiprocurcumenol-Cell invasion protein SipB; 11.Cubebene-Cell invasion protein SipB; 12.IsoprocurcumenolCell invasion protein SipB.

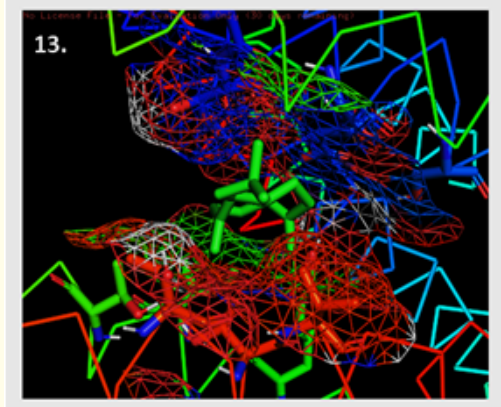

6TUL-Beta-Caryophyllene

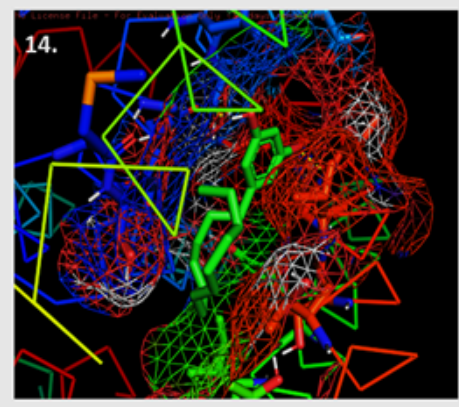

6TUL-1, 3-dihydroxy-5-(tridec-4', 7'dienyl) benzene

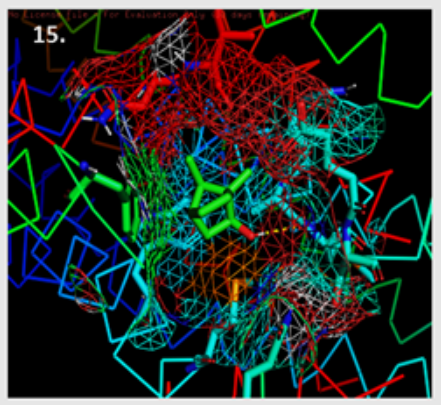

6TUL-DL-Borneol

Figure 3: Mesh presentation of Cell invasion protein SipB (PDB: 6TUL) with top selected compounds. 13. Beta-Caryophyllene- Cell invasion protein SipB; 14. 1, 3-dihydroxy-5-(tridec-4', 7'-dienyl) benzene- Cell invasion protein SipB 15. DL-Borneol -Cell invasion protein SipB. 
Virtual Screening, Molecular Docking, and ADME/T Analysis of Natural Product Library against Cell Invasion Protein SipB from Salmonella enterica serotype typhi: In Silico Analysis

\begin{tabular}{|l|c|c|c|}
\hline Sr. No. & Compounds Name & Pubchem Id & Docking Score (Kcal/mol) \\
\hline 1. & Cyclocurcumin & 69879809 & -8.8 \\
\hline 2. & Stigmasterol & 5280794 & -8.8 \\
\hline 3. & Procurcumenol & 189061 & -8.0 \\
\hline 4. & Curcumenol & 10447050 & -7.8 \\
\hline 5. & 1,7-bis (4-hydroxyphenyl)-1,4,6-heptatrien-3-one & 167812 & -7.7 \\
\hline 6. & iene-3-one & 6437306 & -7.6 \\
\hline 7. & (1E,4E)-1-(4-Hydroxy-3-methoxyphenyl)-5-(4-hydroxyphenyl)-1,4-pentad- & 10469828 & -7.5 \\
\hline 8. & 2-Hepten-4-one, 6-(3-hydroxy-4-methylphenyl)-2-methyl- & 15858385 & -7.3 \\
\hline 9. & E2, 10S)-6, 10-Dimethyl-3-propan-2-ylidenecyclodec-6-ene-1,4-Dione & 6442617 & -7.1 \\
\hline 10. & Cubebene & 10263440 & -7.1 \\
\hline 11. & Isoprocurcumenol & 91747196 & -6.6 \\
\hline 12. & Beta-Caryophyllene & 14543198 & -6.6 \\
\hline 13. & 1,3-dihydroxy-5-(tridec-4',7'-dienyl)benzene & 5281515 & -6.5 \\
\hline 14. & DL-Borneol & 637432 & -6.3 \\
\hline 15. & & 10049 & -5.6 \\
\hline
\end{tabular}

Table 1: Docking score (Kcal/mol) of the cell invasion protein SipB (PDB: 6TUL) with selected compounds detected by molecular docking.

Interactions between the target protein and selected natural compounds

Protein-ligands interactions are generally highlighted by hydrophobic interactions and Hydrogen bonds, which play a specific role in predicting the binding affinity of the ligand with target proteins. All selected fifteen compounds in the dataset were docked into the active site of the Cell Invasion Protein SipB of Salmonella typhi (Figure 4-6) depicts the binding conformation of the selected natural compounds in the binding pocket of the Target protein. The active site of the target protein showed hydrophobic interactions with the help of Val210, Leu101, Lys148, Ile147, Phe129, Ile115, Trp111 and Ala123 etc. amino acids.

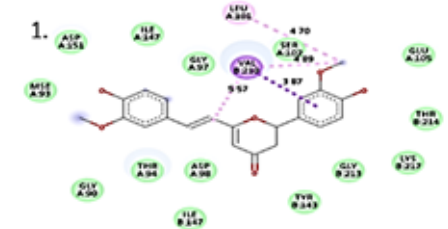

밈

$\square$
2.

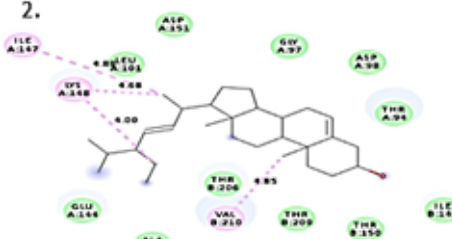

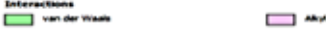

6TUL-Stigmasterol

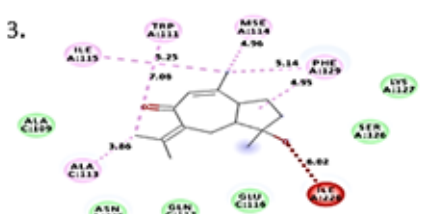

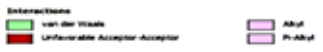

6TUL-Procurcumenol

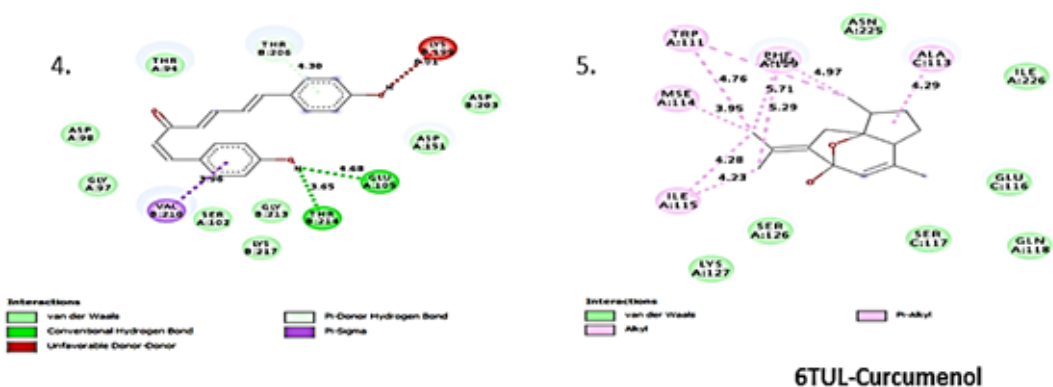

6TUL-1,7-bis (4-hydroxyphenyl)-1,4,6-heptatrien-3-one

Figure 4: Protein-ligand interactions profile of Cell invasion protein SipB (PDB: 6TUL) of Salmonella typhi with selected compounds. 1. Cyclocurcumin-Cell invasion protein SipB; 2. Stigmasterol- Cell invasion protein SipB; 3.Procurcumenol- Cell invasion protein SipB; 4. 1, 7-bis (4-hydroxyphenyl)-1, 4, 6-heptatrien-3-one- Cell invasion protein SipB 5. Curcumenol- Cell invasion protein SipB. 

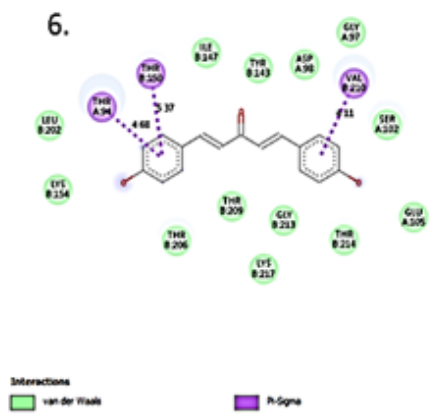

6TUL-1,5-Bis(4-hydroxyphenyl)penta-1,4-dien-3one

9.
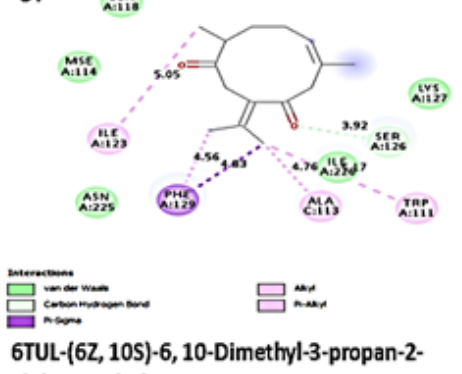

ylidenecyclodec-6-ene-1, 4-Dione
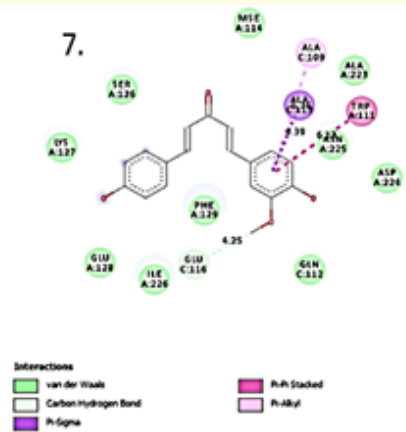

6TUL-(1E,4E)-1-(4-Hydroxy-3-methoxypheny)-5(4-hydroxyphenyl)-1,4-pentadiene-3-one

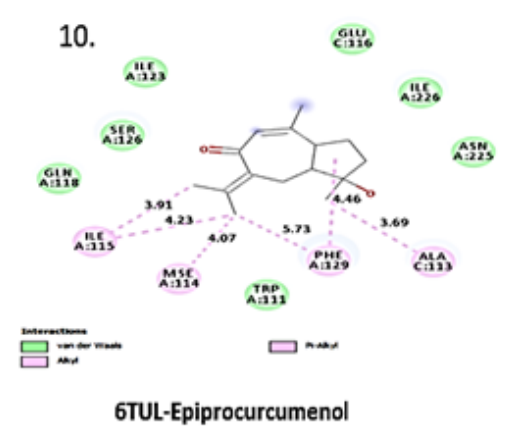

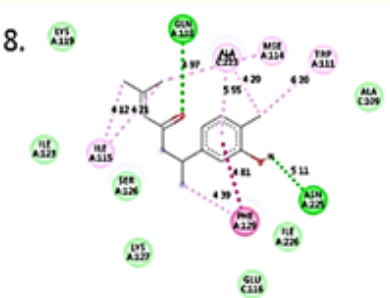

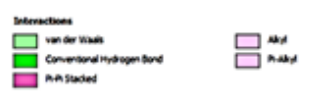

6TUL-2-Hepten-4-one, 6-(3-hydroxy-4methylphenyl)-2-methyl-

Figure 5: Protein-ligand interactions profile of Cell invasion protein SipB (PDB: 6TUL) of Salmonella typhi with selected compounds. 6. 1, 5-Bis (4-hydroxyphenyl) penta-1, 4-dien-3-one-Cell invasion protein SipB; 7. (1E, 4E)-1-(4-Hydroxy-3-methoxyphenyl)-5-(4-hydroxyphenyl)-1, 4-pentadiene-3-one-Cell invasion protein SipB; 8. 2-Hepten-4-one, 6-(3-hydroxy-4-methylphenyl)-2-methyl- Cell invasion protein SipB; 9. 6Z, 10S)-6, 10-Dimethyl-3-propan-2-ylidenecyclodec-6-ene-1, 4-Dione- Cell invasion protein SipB 10. Epiprocurcumenol-Cell invasion protein SipB.

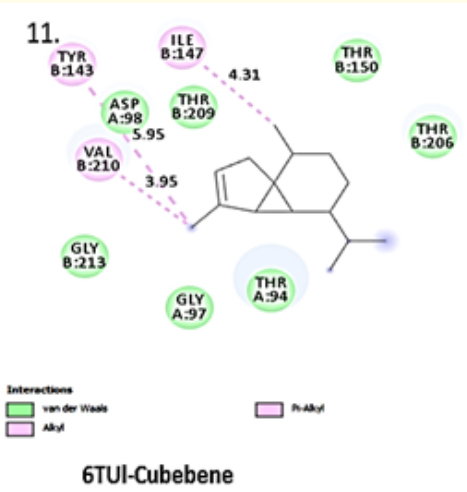

14.

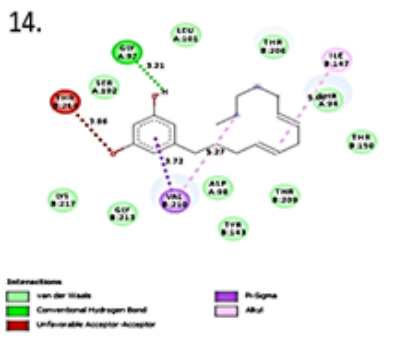

6TUL-1,3-dihydroxy-5-(tridec-4',7'-dienyl)benzene
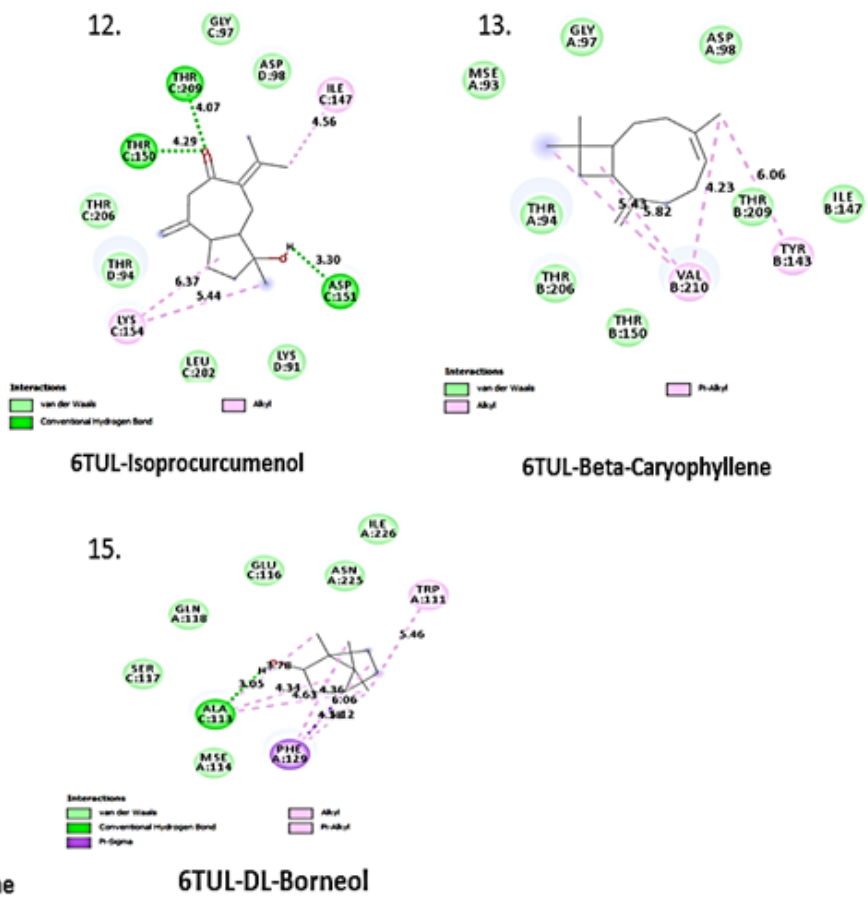

Figure 6: Protein-ligand interactions profile of Cell invasion protein SipB (PDB: 6TUL) of Salmonella typhi with selected compounds. 11.Cubebene-Cell invasion protein SipB; 12.Isoprocurcumenol-Cell invasion protein SipB; 13. Beta-Caryophyllene- Cell invasion protein SipB; 14. 1, 3-dihydroxy-5-(tridec-4', 7'-dienyl) benzene- Cell invasion protein SipB 15. DL-Borneol -Cell invasion protein SipB. 
Virtual Screening, Molecular Docking, and ADME/T Analysis of Natural Product Library against Cell Invasion Protein SipB from Salmonella enterica serotype typhi: In Silico Analysis

The amino acids are present in the active site of Cell Invasion Protein SipB like Val210, Phe129, Ala113, and Trp111 are most common found to create hydrophobic interaction via Pi Hydrophobic, Alkyl Hydrophobic, and mixed Pi/Alkyl Hydrophobic with the top fifteen natural ligands. Beside hydrophobic interactions, there are some hydrogen bonds present between amino acids and top natural compounds. The active site of the Cell Invasion protein SipB showed hydrogen bonds with the help of Thr214, Glu105, Glu116, Gln118, Asn225, Ser126, Asp151, Thr150, Thr209, Gly97, and Ala113 amino acids (Table 2).

\begin{tabular}{|c|c|c|c|}
\hline Sr. No. & Compounds Name & Hydrophobic Interactions & Hydrogen Bonds \\
\hline 1. & Cyclocurcumin & Val B:210, Leu A:101 & - \\
\hline 2. & Stigmasterol & Val B:210, Lys A:148, Ile A:147 & - \\
\hline 3. & Procurcumenol & $\begin{array}{l}\text { Ala C:113, Ile A:115, Trp A:111, } \\
\text { Mse A:114, Phe A:129 }\end{array}$ & - \\
\hline 4. & 1,7-bis (4-hydroxyphenyl)-1,4,6-heptatrien-3-one & Val B:210 & Thr B: 214, Glu A:105 \\
\hline 5. & Curcumenol & $\begin{array}{c}\text { Ile A:115, Mse A:114, } \\
\text { Trp A:111, Phe A:129, } \\
\text { Ala C:113 }\end{array}$ & - \\
\hline 6. & 1,5-Bis(4-hydroxyphenyl)penta-1,4-dien-3-one & Thr A:94, Thr B:150, Val B:210 & - \\
\hline 7. & $\begin{array}{l}\text { (1E,4E)-1-(4-Hydroxy-3-methoxyphenyl)-5-(4- } \\
\text { hydroxyphenyl)-1,4-pentadiene-3-one }\end{array}$ & $\begin{array}{l}\text { Ala C: } 109, \text { Ala C: } 113 \\
\text { Trp A: } 111\end{array}$ & Glu C:116 \\
\hline 8. & 2-Hepten-4-one, 6-(3-hydroxy-4-methylphenyl)-2-methyl- & $\begin{array}{c}\text { Ile A:115, Phe A:129, } \\
\text { Ala C: } 113, \text { Mse A:114, Trp A:111 }\end{array}$ & Gln A: 118, Asn A:225 \\
\hline 9. & $\begin{array}{c}(6 \mathrm{Z}, 10 \mathrm{~S})-6,10 \text {-Dimethyl-3-propan-2-ylidenecyclodec-6-ene-1, } \\
\text { 4-Dione }\end{array}$ & $\begin{array}{l}\text { Ile A:123, Phe A:129, } \\
\text { Ala C:113, Trp A:111 }\end{array}$ & Ser A:126 \\
\hline 10. & Epiprocurcumenol & $\begin{array}{l}\text { Ile A:115, Mse A:114, } \\
\text { Phe A:129, Ala C:113 }\end{array}$ & - \\
\hline 11. & Cubebene & Val B:210, Tyr B:143, Ile B:147 & - \\
\hline 12. & Isoprocurcumenol & Ile C:147, Lys C:154 & $\begin{array}{l}\text { Asp C:151, Thr C:150, } \\
\text { Thr C: } 209\end{array}$ \\
\hline 13. & Beta-Caryophyllene & Val B:210, Tyr B:143 & - \\
\hline 14. & 1,3-dihydroxy-5-(tridec-4',7'-dienyl)benzene & Ile B: 147, Val B:210 & Gly A:97 \\
\hline 15. & DL-Borneol & Trp A:111 & Ala C:113 \\
\hline
\end{tabular}

Table 2: Protein-ligand interaction profile of cell invasion protein SipB of Salmonella typhi (PDB: 6TUL) with selected compounds.

A good number of amino acids are found to appear in the hydrogen bonding and hydrophobic interactions. The active site of the Cell Invasion Protein SipB has mostly show hydrophobic interactions with the top ligands. Hydrophobic interaction successfully delineates specific functional groups that may be responsible for the hydrophobic generating effect of these compounds with strong binding affinity against target Cell Invasion Protein SipB and may play a highly influencing against Salmonella enterica serovar Typhi infection.
ADMET properties of leads compounds

ADME/T properties of the lead compounds (Figure 4-6) were appraised by using the DruLiTo is an open source software. It can calculate different molecular properties and screen the molecules based on drug likeness rules such as, Lipinski's rule, MDDR-like rule, Veber Rule, Ghose Filter, BBB Rule, and CMC-50 like rule and Quantitative estimate of drug-likeness (QED) (Figure 8 and table 3). Swiss ADME BOILEDegg (Figure 7) allows for evaluation of HIA as a function of the position of the fifteen molecules in the WLOGP- 
Versus-TPSA referential. Moreover, polar surface area, high oral bioavailability, Hbond donors, and acceptors are being important criteria for the development of therapeutic agents. Molecule 1 (Cyclocurcumin) is predicted as absorbed by gastro intestines (White Region) but are not brain penetrate (Yolk) (Figure 7). All the molecules evaluated are PGP- not subject to active efflux (Red dot) (Figure 7) All these models unscrew the qualitative prediction and ranking of absorption, for- mulation effects on drug permeability, determining the mechanism(s) of permeability, and the potential for transporter-mediated drug-drug interactions. All the good scoring ligand has drug-likeness properties by Lipinski's rule. It may also be predictable that the combination of these drugs/s may be a good idea to prevent Salmonella enterica serovar Typhi infection.

\begin{tabular}{|c|c|c|c|c|c|c|c|c|c|c|c|c|c|c|c|c|c|}
\hline $\begin{array}{l}\text { Sr. } \\
\text { No. }\end{array}$ & Molecule Name & $\begin{array}{c}\text { Com- } \\
\text { pound Id }\end{array}$ & Mw & $\log P$ & Alogp & HBA & HBD & TPSA & AMR & nRB & $\begin{array}{c}\text { nAtom } \\
\text { Ring }\end{array}$ & $\begin{array}{l}\text { nAcid- } \\
\text { ic }\end{array}$ & RC & $\begin{array}{c}\text { nRig } \\
\text { idB }\end{array}$ & nArom & nHB & $\begin{array}{c}\text { S } \\
\text { Alerts }\end{array}$ \\
\hline 1. & Cyclocurcumin & 69879809 & 368.13 & 1.895 & 0.241 & 6 & 2 & 85.22 & 110.77 & 5 & 47 & 0 & 3 & 24 & 2 & 8 & 1 \\
\hline 2. & Stigmasterol & 5280794 & 412.37 & 11.017 & 1.257 & 1 & 1 & 20.23 & 125.29 & 5 & 78 & 0 & 4 & 28 & 0 & 2 & 1 \\
\hline 3. & Procurcumenol & 189061 & 234.16 & 1.671 & 2.091 & 2 & 1 & 37.3 & 69.39 & 0 & 39 & 0 & 2 & 18 & 0 & 3 & 1 \\
\hline 4. & $\begin{array}{c}\text { 1,7-bis } \\
\text { (4-hydroxyphenyl)- } \\
\text { 1,4,6-heptatrien- } \\
\text { 3-one }\end{array}$ & 10447050 & 292.11 & 2.498 & 2.216 & 3 & 2 & 57.53 & 98.83 & 5 & 38 & 0 & 2 & 18 & 2 & 5 & 2 \\
\hline 5. & Curcumenol & 167812 & 234.16 & 1.748 & 2.327 & 2 & 1 & 29.46 & 86.04 & 0 & 39 & 0 & 3 & 19 & 0 & 3 & 1 \\
\hline 6. & $\begin{array}{l}\text { 1,5-Bis(4-hydroxy- } \\
\text { phenyl)penta-1,4- } \\
\text { dien-3-one }\end{array}$ & 6437306 & & & & & & & & & & & & & & & \\
\hline 7. & $\begin{array}{c}\text { (1E,4E)-1-(4- } \\
\text { Hydroxy-3- } \\
\text { methoxyphenyl)-5- } \\
\text { (4-hydroxyphenyl)- } \\
\text { 1,4-pentadiene-3- } \\
\text { one }\end{array}$ & 10469828 & 296.10 & 1.833 & 1.246 & 4 & 2 & 66.67 & 95.16 & 5 & 38 & 0 & 2 & 18 & 2 & 6 & 1 \\
\hline 8. & $\begin{array}{c}\text { 2-Hepten-4-one, } \\
\text { 6-(3-hydroxy- } \\
\text { 4-methylphenyl)- } \\
\text { 2-methyl- }\end{array}$ & 15858385 & 232.15 & 2.322 & 2.445 & 2 & 1 & 37.30 & 74.08 & 4 & 37 & 0 & 1 & 13 & 1 & 3 & 1 \\
\hline 9. & $\begin{array}{l}\text { (6Z,10S)-6, 10-Di- } \\
\text { methyl-3-propan- } \\
\text { 2-ylidenecyclodec- } \\
\text { 6-ene-1, 4-Dione }\end{array}$ & 6442617 & 234.16 & 2.039 & 2.623 & 2 & 0 & 34.14 & 71.53 & 0 & 39 & 0 & 1 & 17 & 0 & 2 & 2 \\
\hline 10. & Epiprocurcumenol & 10263440 & 234.16 & 1.671 & 2.091 & 2 & 1 & 37.30 & 69.39 & 0 & 39 & 0 & 2 & 18 & 0 & 3 & 1 \\
\hline 11. & Cubebene & 91747196 & 204.19 & 6.499 & 1.645 & 0 & 0 & 0.00 & 63.99 & 1 & 39 & 0 & 3 & 16 & 0 & 0 & 1 \\
\hline 12. & Isoprocurcumenol & 14543198 & 234.16 & 1.56 & 1.83 & 2 & 1 & 37.30 & 68.34 & 0 & 39 & 0 & 2 & 18 & 0 & 3 & 2 \\
\hline 13. & Beta-Caryophyllene & 5281515 & 204.19 & 6.044 & 3.229 & 0 & 0 & 0.00 & 67.69 & 0 & 39 & 0 & 2 & 16 & 0 & 0 & 1 \\
\hline 14. & $\begin{array}{l}\text { 1,3-dihydroxy- } \\
\text { 5-(tridec-4',7'-die- } \\
\text { nyl)benzene }\end{array}$ & 637432 & 288.21 & 6.926 & 0.552 & 2 & 2 & 40.46 & 89.76 & 10 & 49 & 0 & 1 & 11 & 1 & 4 & 2 \\
\hline 15. & DL-Borneol & 10049 & 154.14 & 2.734 & 0.943 & 1 & 1 & 20.23 & 44.28 & 0 & 29 & 0 & 2 & 12 & 0 & 2 & 0 \\
\hline
\end{tabular}

Table 3: Structural; physicochemical; biochemical; pharmacokinetics and toxicity properties of top fifteen natural compounds.

MW: Molecular Weight; Accept H: Estimated number of hydrogen bonds that would be accepted by the solute from water molecules in an aqueous solution; Donor H: Estimated number of hydrogen bonds that would be donated by the solute to water molecules in an aqueous solution; TPSA (Total Polar Surface Area: PSA is a reference point for since the latter descriptor is a ratio of the (Lipophilicity to hydrophilicity AlogP98); No of Rigid Bonds $>=18$; Number of Rings $>=3$; Number Rotable Bonds $>=6$;

In Ghose Filter (MW between 160 to 480, LogP value between -0.4 to 5.6, Atom Count between 20 to 70, Refractivity between 40 to 130); BBB Likeness property (Mw<=400, H-bonds total <=8, No acids =0); CMC-50 Lik Rule (LogP value between 1.3-4.1, Atom count between 30 to 55, Mw between 230 to 390 and Molar Refractivity between 70 to 110); Unweighted QED ( Weighted QED $>=0.5$, Unweighted QED $>=0.5$ ); Veber Rule (Rotable Bond $<=10$, Polar Surface Area $<=140$ ). 


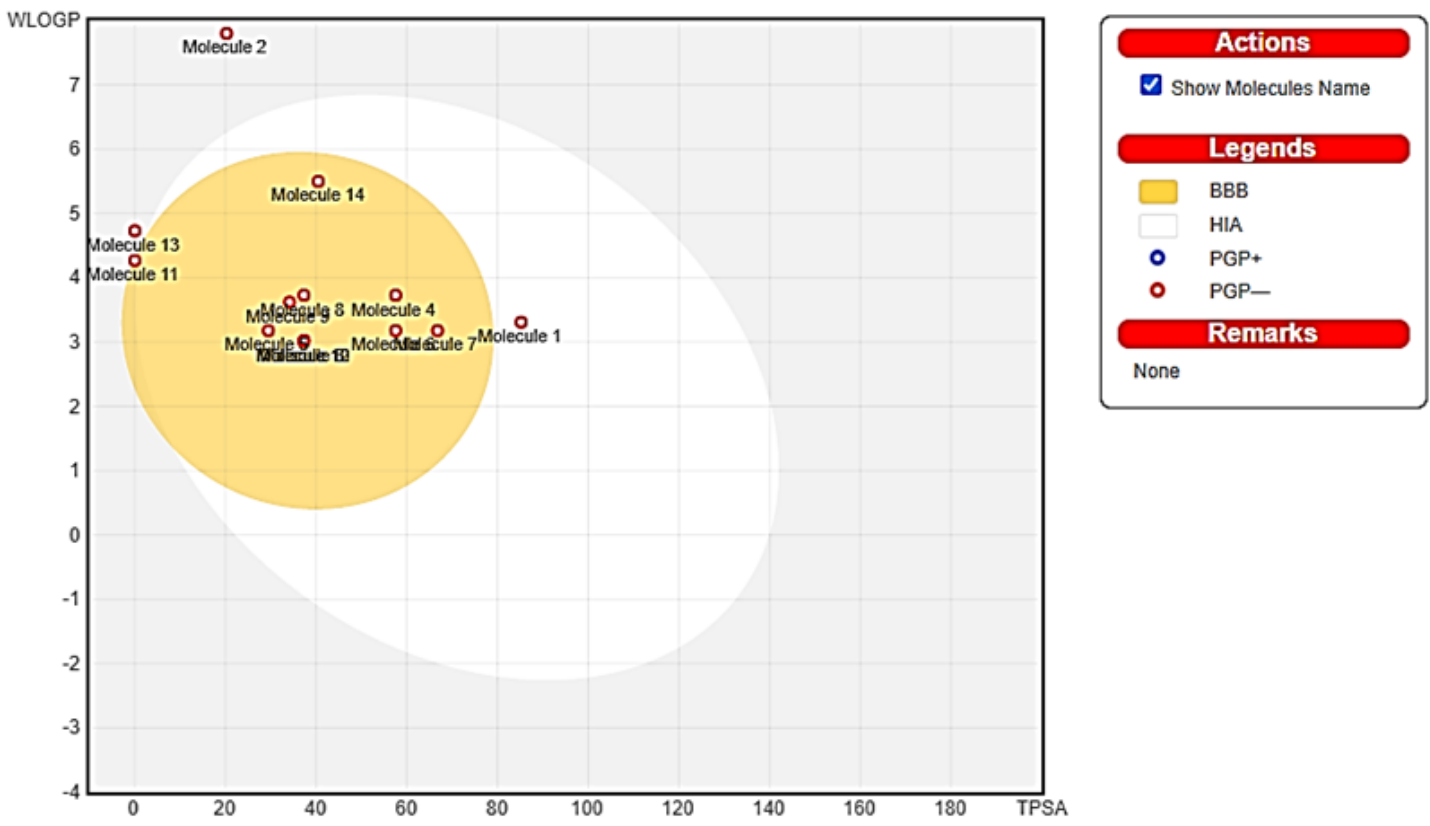

Figure 7: Boiled egg diagram and bioavailability rader map of top fifteen natural compounds.

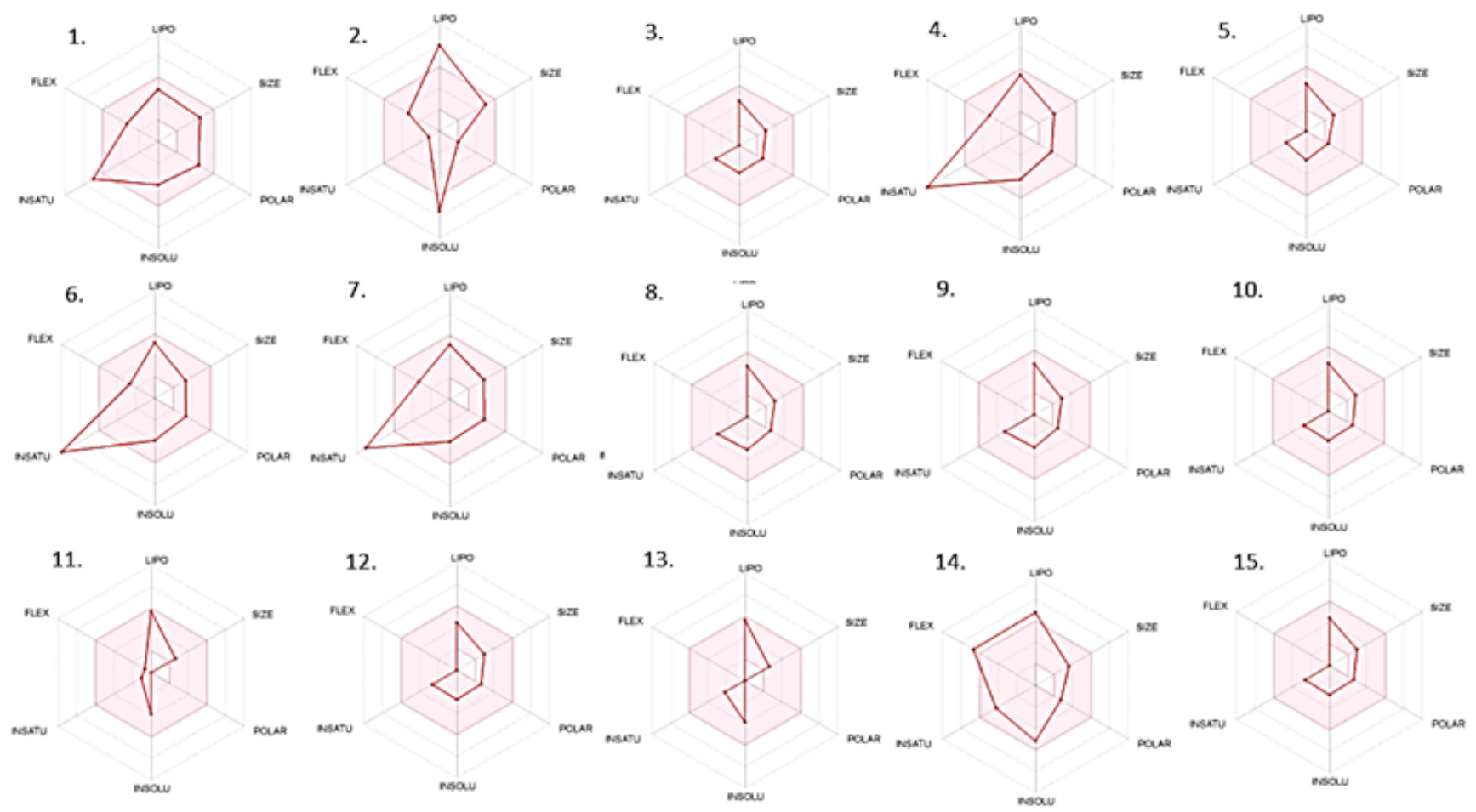

Figure 8: All the fifteen compounds show in colored zone are the suitable physiochemical space for oral bioavailability and show the LIPO(Lipophilicity), SIZE (Molecular Weight), POLAR (Polarity), INSOLU (Insolubility), INSATU (Instauration) and FLIX (Rotable bond flexibility) parameters. 


\section{Conclusion}

In this study, it has been shown that selected fifteen natural screened compounds have good docking score and strong hydrophobic interactions and Some H-bonding and follows the Lipinski rule of five. Thus, these compounds might have the potential to be utilized against the Salmonella enterica serovar typhi infection. Here, in this study, we can conclude that the obtained compounds from this In Silico study may be an excellent agent to combat Salmonella enterica serovar typhi infection either alone or in combination. However, the natural product library available in the form of solution and the evaluation of their in vitro and in vivo activity against Cell invasion protein SipB could be an interesting target for the development of new antibiotic /drugs for the near future applications for treatment and follow-up of the patients as well.

\section{Acknowledgement}

We thanks to those who are directly or indirectly helping us to successful in this study. I heartily congratulate to my father Durga Lal Verma and my Mother Kali Devi and Sisters for their extensive support. We deeply understand and thanks to our institution Mewar University and respective department are merely acknowledgeable. We highly thankful to use the software's (RBPS Web Portal, BIOVIA Company Discovery studio 2020, PyRx Software, PyMOL, and Swiss institute of Bioinformatics).

\section{Conflicts of Interest}

The authors declare no conflict of interest.

\section{Bibliography}

1. Alcaine SD., et al. "Antimicrobial resistance in nontyphoidal Salmonella". Journal of Food Protection 70.3 (2007): 780-790.

2. Álvarez-Fernández E., et al. "Prevalence and antimicrobial resistance of Salmonella serotypes isolated from poultry in Spain: Comparison between 1993 and 2006". International Journal of Food Microbiology 153.3 (2012): 281-287.

3. Borsoi A., et al. "Behavior of Salmonella heidelberg and Salmonella enteritidis strains following broiler chick inoculation: Evaluation of cecal morphometry, liver and cecum bacterial counts and fecal excretion patterns". Brazilian Journal of Microbiology 42.1 (2011): 266-273.

4. Eng SK., et al. "Salmonella: A review on pathogenesis, epidemiology and antibiotic resistance". Frontiers in Life Science 8.3 (2015): 284-293.
5. Majowicz SE., et al. "The Global Burden of Nontyphoidal Salmonella Gastroenteritis". Clinical Infectious Diseases 50.6 (2010): 882-889.

6. Goay YX., et al. "Identification of Five Novel Salmonella typhiSpecific Genes as Markers for Diagnosis of Typhoid Fever Using Single-Gene Target PCR Assays". BioMed Research International (2016).

7. TC Darton., et al. "Typhoid epidemiology, diagnostics and the human challenge model”. Current Opinion in Gastroenterology 30 (2014): 7-17.

8. Ziech RE., et al. "Multidrug resistance and ESBL-producing Salmonella spp. isolated from broiler processing plants". Brazilian Journal of Microbiology 47.1 (2016): 191-195.

9. Penha Filho RAC., et al. "Antimicrobial susceptibility of Salmonella gallinarum and Salmonella pullorum isolated from ill poultry in brazil". Ciencia Rural 46.3 (2016): 513-518.

10. Shivaprasad HL. "Fowl typhoid and pullorum disease". OIE Revue Scientifique et Technique 19.2 (2000): 405-424.

11. Guibourdenche M., et al. "Supplement 2003-2007 (No. 47) to the White-Kauffmann-Le Minor scheme". Research in Microbiology 161.1 (2010): 26-29.

12. LL M., et al. "Supplement $1989\left(\mathrm{n}^{\circ}\right.$ 33) to the kauffmann-white scheme”. Research in Microbiology 141.9 (1990): 1173-1177.

13. Suresh X. "In silico studies on structure prediction and inhibitory action of selected natural compounds on cell invasion protein SipB from Salmonella Typhi". Asian Journal of Biomedical and Pharmaceutical Sciences 4.32 (2014): 20-24.

14. Kaur J and Jain SK. "Role of antigens and virulence factors of Salmonella enterica serovar Typhi in its pathogenesis". Microbiological Research 167.4 (2012): 199-210.

15. Galán JE and Collmer A. “Type III secretion machines: Bacterial devices for protein delivery into host cells". Science 284.5418 (1999): 1322-1328.

16. Calixto JB. "The role of natural products in modern drug discovery". Anais da Academia Brasileira de Ciências (2016): 91.

17. Pilot P. "Dassault Systèmes BIOVIA, discovery studio modelling environment" (2016). 
18. Morris GM., et al. "Automated docking using a Lamarckian genetic algorithm and an empirical binding free energy function". Journal of Computational Chemistry 19.14 (1998): 1639-1662.

\section{Assets from publication with us}

- Prompt Acknowledgement after receiving the article

- Thorough Double blinded peer review

- Rapid Publication

- Issue of Publication Certificate

- High visibility of your Published work

Website: www.actascientific.com/

Submit Article: www.actascientific.com/submission.php

Email us: editor@actascientific.com

Contact us: +919182824667 